\title{
Least square regularization inversion of transient electromagnetic method
}

\author{
Fengping $\mathrm{Li}^{1, \mathrm{a}}$, Haiyan Yang ${ }^{1, \mathrm{~b}}$ \\ ${ }^{1}$ State Key Laboratory of Nuclear Resources and Environment, East China University of Technology, Nan Chang, China \\ ageophysics_Lee@163.com, benious_yang@126.com
}

\begin{abstract}
The traditional optimization inversion method based on iterative method strongly depends on the selection of the initial model, which may easily leads to the local optimal solution, however, the smoke ring inversion has the advantages of fast calculation and no need of initial model. Therefore, the results of smoke ring inversion were proposed as the initial model, and the Tikhonov's regularization thought was applied to the least square optimization inversion method, then the least square regularization inversion objective function of smoothest model constraints was obtained. Finally, the automatic iterative inversion for one-dimensional layered model of transient electromagnetic method was carried out. the inversion results of high precision were obtained, which proved that the least square regularization inversion algorithm of transient electromagnetic method is effective and feasible.
\end{abstract}

Keywords - transient electromagnetic method; smoothest model constraints;smoke ring inversion;least square regularization inversion

\section{INTRODUCTION}

The inversion of transient electromagnetic method(TEM for short) now mostly stay in the one-dimensional inversion stage, two or three dimensional inversion method is still in the research stage or applied in a special way, the study of onedimensional inversion meet higher accuracy requirements remains current urgent problems in the application of transient electromagnetic sounding. At present, the most commonly used traditional optimization inversion method strongly depends on the choice of the initial model, if the selection of initial model is not appropriate, it is easy to fall into the local optimal solution. In this paper, results of smoke ring inversion $^{[1]}$ which does not require initial model were taken as the initial model, and the least square regularization method ${ }^{[2]}$ is used to achieve a higher accuracy of the one dimensional transient electromagnetic automatic iterative inversion.

\section{FORWARD}

The Forward modeling is the basis of inversion, the integral of Bessel function ${ }^{[3]}$ was calculated with the Fast Hankel transform of D.Guptasarma and B.Singh, and using the improved cosine transform numerical filtering method ${ }^{[4]}$ to calculate the cosine integral, thus the response of horizontal layered earth electromagnetic under excitation of the step current was calculated. By using the binary search method ${ }^{[5]}$, the all-time apparent resistivity was calculated.

\section{INVERSION}

\section{A. Least Square Regularization Inversion}

The inverse problem of transient electromagnetic method is usually ill-posed, and the results obtained are non-unique. In order to improve the stability and non-uniqueness of the solution, the regularization thought of Tikhonov was introduced

$$
P^{\alpha}(m)=\varphi(m)+\alpha S(m)
$$

Where $P^{\alpha}(m)$ is the total objective function, $\alpha$ is a regularization parameter which controls the trade-off between these two contributions in this minimization process, $\varphi(m)$ is a misfit function, $S(m)$ is a stabilizing function. The smoothest model constraints based on a priori model is used here, then the total objective function of the transient electromagnetic inversion problem can be expressed as

$$
P^{\alpha}(m)=\left\|W_{d}\left[d^{o b s}-F(m)\right]\right\|^{2}+\alpha\left\|W_{m}\left(m-m_{0}\right)\right\|^{2}
$$

Where $F$ is a forward modeling operator which is generally non-linear, $W_{d}$ is the weight coefficient matrix of observation data, $W_{m}$ is the weight coefficient matrix of model, $m_{0}$ is prior model. The iterative form of the least square regularization inversion with the constraint inversion condition is

$$
\begin{aligned}
& \Delta m=\left[\left(J^{k T} W_{d}^{T} W_{d} J^{k}+\alpha W_{m}{ }^{T} W_{m}\right)\right]^{-1} . \\
& {\left[J^{k T} W_{d}{ }^{T} W_{d} J^{k}\left(d^{o b s}-d^{k}\right)+\alpha W_{m}^{T} W_{m}\left(m_{0}-m^{k}\right)\right]}
\end{aligned}
$$

Where $m^{k}$ is the k-th iteration value of the model, $J^{k}$ is the Jacobi sensitivity matrix.

\section{B. Sensitivity Matrix Calculation}

The calculation of sensitivity matrix in geophysical inversion is an important link in the inversion calculation. For the one dimensional inversion problems of transient electromagnetic sounding, perturbation method can be used to calculate the sensitivity matrix, namely the use of a first-order finite difference formula (4) to calculate their approximate 
disturbance on the k-th parameter $\Delta m_{k}$, and through forward calculation to obtain the transient electromagnetic response after the disturbance.

$$
\frac{\partial F_{j}(m)}{\partial m_{k}} \approx \frac{F_{j}\left(m+\Delta m_{k}\right)-F_{j}(m)}{\Delta m_{k}}
$$

\section{Inversion Scheme}

\section{i). Model ParameterDescription}

For the one-dimensional transient electromagnetic layered model, the model parameters $m$ can be expressed as

$$
m=\left(\rho_{1}, \rho_{2}, \cdots, \rho_{N}, h_{1}, h_{2}, \cdots, h_{N-1}\right)
$$

In addition, when the thickness of each layer is certain, only the resistivity is the real inversion parameter, so that the model parameters $m$ can be expressed as

$$
m=\left(\rho_{1}, \rho_{2}, \cdots, \rho_{M}\right)
$$

Where, $M>N$ ( $N$ is the layer parameters of actual geoelectric model).In the calculation of one dimensional inversion, the data object function can be fitted to the all-time apparent resistivity, which can be expressed as

$$
\Phi_{d}=\sum_{i=1}^{n}\left(\frac{\rho_{o b s(i)}-\rho_{f(i)}}{\rho_{o b s(i)}}\right)^{2}
$$

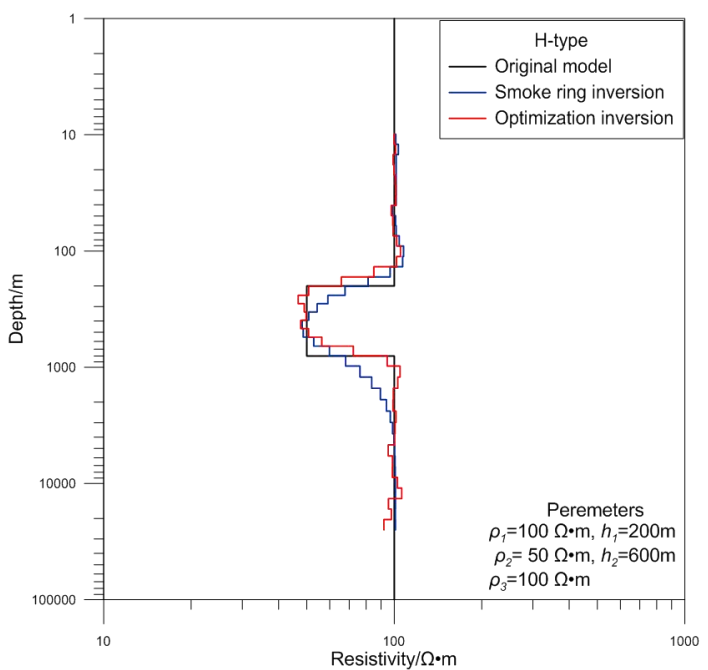

Fig.1. Inversion result of H-type geoelectric model
Where, $\rho_{\text {obs }(i)}$ and $\rho_{f(i)}$ are all-time apparent resistivity of the measured data and model response separately, $n$ is the sample frequency points.

ii). Selection of Initial Model

The inversion results of the smoke ring inversion which proposed by Jiang Bangyuan according to M.N.Nabighian's derivation can be used as the initial model parameters. The results of the smoke ring inversion are not accurate enough, but it has the advantages of no need of initial model and simple calculation, and can directly give the change form of underground resistivity with depth. Here remain layer thickness of smoke ring inversion results fixed, only the inversion of resistivity parameters.

\section{Simulation Examples}

In order to test the effectiveness and feasibility of the above algorithm, the inversion calculation of H-type and K-type geoelectric model of three layers and $\mathrm{KH}$-type geoelectric model of four layers were carried out. Parameters of H-type were set to: resistivity $\rho_{1}=100 \Omega \cdot \mathrm{m}, \rho_{2}=50 \Omega \cdot \mathrm{m}, \rho_{3}=100 \Omega \cdot \mathrm{m}$, thickness $\mathrm{h}_{1}=200 \mathrm{~m}, \mathrm{~h}_{2}=600 \mathrm{~m}$; Parameters of K-type is set to:resistivity $\rho_{1}=50 \Omega \cdot \mathrm{m}, \rho_{2}=100 \Omega \cdot \mathrm{m}, \rho_{3}=50 \Omega \cdot \mathrm{m}$, thickness $\mathrm{h}_{1}=200 \mathrm{~m}, \mathrm{~h}_{2}=600 \mathrm{~m}$; Parameters of KH-type were set to: resistivity $\rho_{1}=100 \Omega \cdot \mathrm{m}, \rho_{2}=200 \Omega \cdot \mathrm{m}, \rho_{3}=50 \Omega \cdot \mathrm{m}, \rho_{4}=200 \Omega \cdot \mathrm{m}$, thickness $\mathrm{h}_{1}=100 \mathrm{~m}, \mathrm{~h}_{2}=400 \mathrm{~m}, \mathrm{~h}_{3}=900 \mathrm{~m}$. In the inversion calculation, the smoke ring inversion was first carried out for observation data to obtain layer thickness of geoelectric model which remain fixed over the next whole inversion process, only the inversion of resistivity parameters. The calculated depth-resistivity sections were shown below.

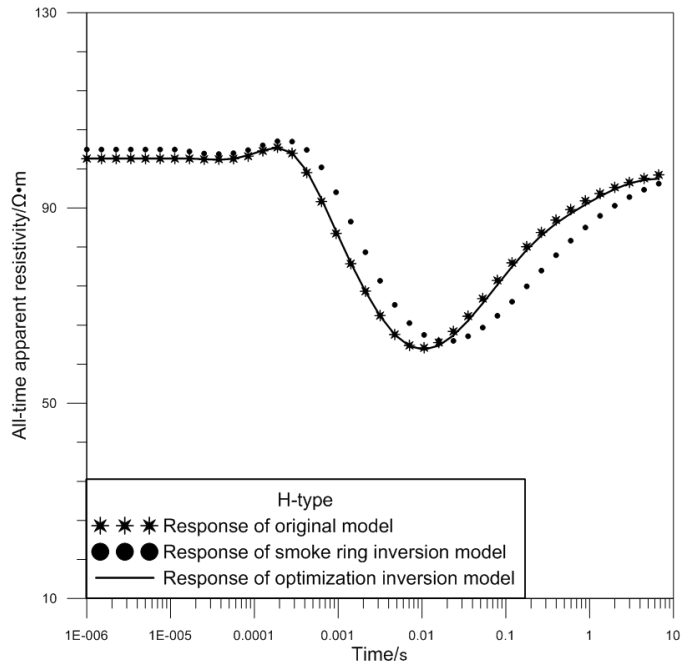

Fig.2.Response of H-type geoelectric inversion model 


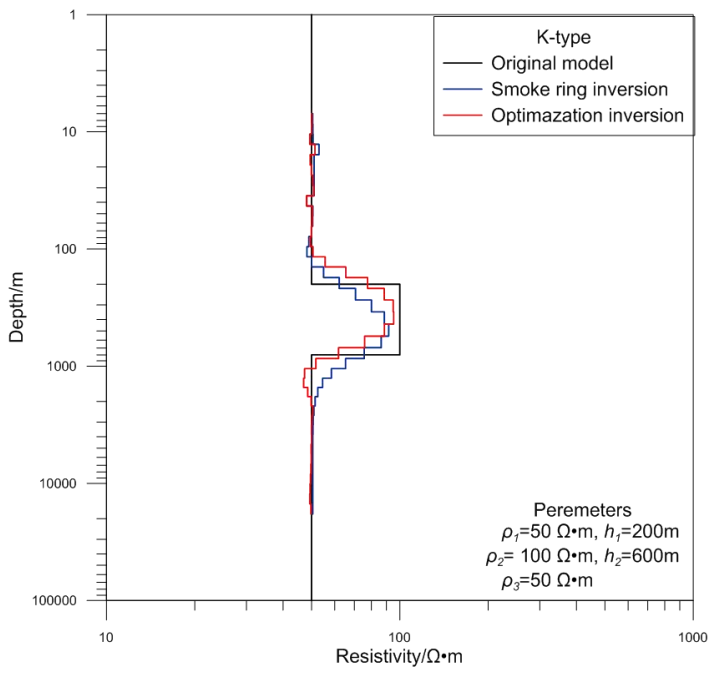

Fig.3.Inversion result of K-type geoelectric model

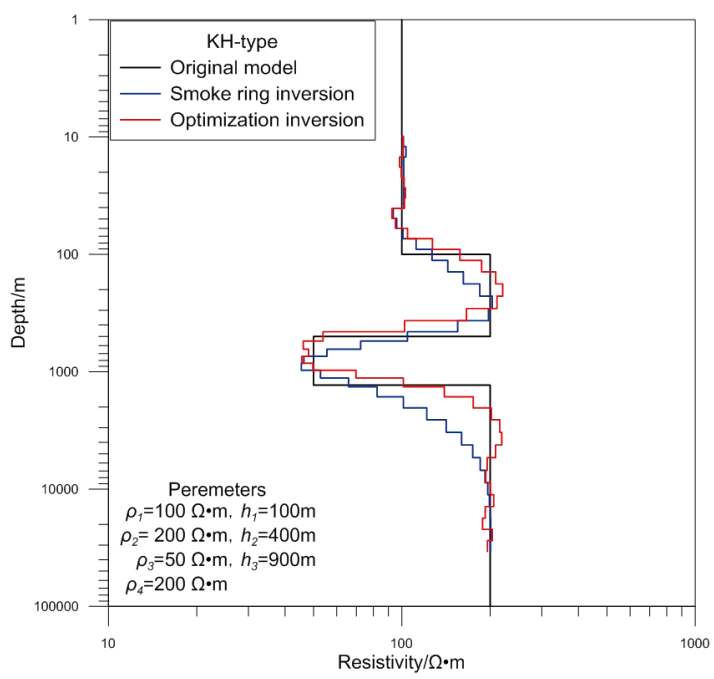

Fig.5.Inversion result of KH-type geoelectric model

As can be seen from Fig. 1,3,5, the inversion results agree well with the actual geoelectric model, compared with the smoke ring inversion, the results of least squares regularization inversion more accurately reflect the size and location of the high and low resistivity layer. From the perspective of data fitting (Fig. 2,4,6), the response of onedimensional least square regularization inversion results fit the theoretical data very well, better than the fitting curves of smoke ring inversion.

\section{CONCLUSION}

1) Application of Fast Hankel transform, improved cosine transform numerical filtering method to the high precision forward of one-dimensional transient electromagnetic method, the binary search method can quickly calculate the all-time apparent resistivity, The results show that the forward modeling program is correct.

2) Using least square regularization inversion algorithm for the forward and inverse calculation of the typical

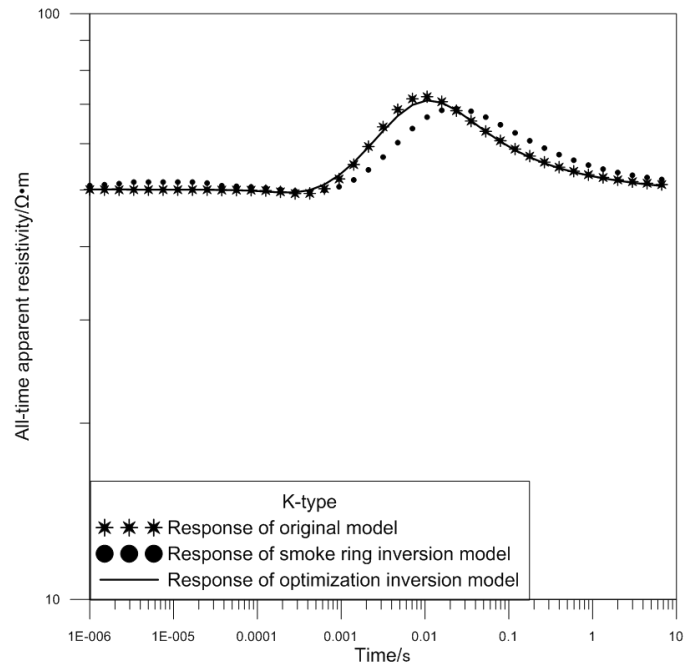

Fig.4.Response of K-type geoelectric inversion model

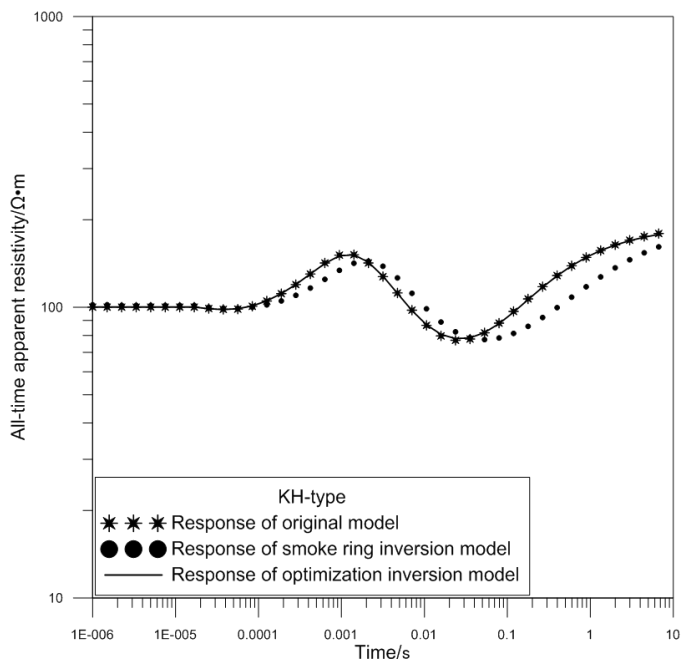

Fig.6. Response of KH-type geoelectric inversion model

geoelectric model, due to the adoption of the smoothest constrained model objective function, situation of transient electromagnetic method which was not sensitive enough to high resistivity layer has been improved. What's more, for the complex geological structure, this method also has good effectiveness and adaptability.

3) Iterative inversion of about 10 times will be able to stabilize the convergence, and get satisfactory results, verify the method is feasible for data interpretation of onedimensional transient electromagnetic method.

\section{ACKNOWLEDGMENT}

Thanks to my tutor Haiyan Yang for providing support and useful guidance. This study gains the bankroll of the National Natural Science Foundation of China (Grant No.41564001) and the Natural Science Foundation of Jiangxi Province (Grant No: 20151BAB203045). 


\section{REFERENCES}

[1] Bangyuan Jiang. Applied near zone magnetic source transient electromagnetic exploration[M],Geological Publishing House. 1998.

[2] Jian-xin Liu, Xiao-zhong Tong, Rong-wen Guo,ect. Magnetotelluric sounding exploration: Data processing inversion and interpretation [M].Beijing: Science Press. 2012.
[3] Guptasarma, B.Singh. New digital linear filters for Hankel J0 and J1 transforms [J]. Geophysical Prospecting [J],45: 745-762.1997.

[4] Huajun Wang. Digital filtering algorithm of sine and cosine transform [J]. Engineering Geophysics, 51(6):1936 1942. 2004.

[5] Qingli Chen. binary search algorithm for the all time apparent resistivity of Transient Electromagnetic Method [J], Journal of Oil and Gas Technology.31 (02): 45-49. 2009. 